\title{
Evidence based mammography screening: is evidence changing?
}

\author{
U. A Liyanage, U. M. J. E Samaranayake \\ Faculty of Medicine, University of Colombo, Sri Lanka
}

Keywords: Breast cancer; mammography; population screening

\begin{abstract}
Population-based mammography screening programmes were launched by developed countries in the latter half of the twentieth century based on the promising evidence on mortality benefits reported by the earliest randomised control trials (RCTs). It was widely accepted that screening mammography significantly reduced breast cancer mortality in women invited for screening. However, since the first RCT in the 1960s, varying evidence has been tabled by many researchers, sometimes alarming the public, drawing attention to the harms of screening mammography, mainly identified as overdiagnosis and false positives. While overtreatment is recognised as a consequence of over diagnosis, a relatively newer set of harms categorized under psychological distress have been highlighted as resultant from false positive screening tests. Currently, the magnitude of the harms of screening mammography remains uncertain. The most turbulent reviews on breast screening, however, were the queries into the well and long believed mortality benefits of mammography screening first tabled in the 1970s by the Canadian national breast screening study. A Cochrane review by Gøtzsche and Jørgensen further added to the negative attributes of mammography screening as they concluded that the actual current mortality benefits are nonsignificant and smaller than that claimed by many RCTs which were poorly randomised. Although heavily criticized, sparks ignited by these reports have led many countries worldwide to conduct their panel discussions on evidence on population screening mammography. While many countries have decided to continue their programmes understanding the harms involved, Switzerland in this light has discontinued their population-based mammography screening programme. The debate continues; currently while not overlooking the mortality benefits of mammography, it is also understood that the mortality benefits are not solely due to mammography but are also contributed by other factors such as advancement in
\end{abstract}

Correspondence: U.A. Liyanage

E-mail: udari@anat.cmb.ac.lk

Received: 13-06-2019 Accepted: 17-07-2019

(iD) http://orcid.org/0000-0003-2065-7010

DOI: http://doi.org/10.4038/sljs.v37i2.8625 therapeutic measures and awareness on breast cancer created among the females. In this regard, critical evaluation of global literature is imperative to make country-specific policy decisions for middle-income countries such as Sri Lanka, who are contemplating the options for expanding opportunistic mammography screening services to reach wider population screening targets.

\section{Introduction}

In the latter half of the 20th century, many developed countries invested in population-based mammography breast cancer screening programmes with varying national policies between countries. Many middle-income countries, not investing in expensive organized national programmes, went on to offer mammography as an opportunistic screening method for breast cancer. Embarking on mass screening mammography occurred as recommended by world's leading guideline groups, largely supported by the scientific evidence which was tabled at that time highlighting the mortality benefits of screening mammography. It is not an exaggeration to state that screening mammography has drawn more global attention than any other cancer-screening programme, subject to intense scrutiny over the last few decades [1]. Since the first randomized trial in the early 1960s, variable evidence has been tabled on harms against benefits of screening mammography. Recently, some authors have even challenged the earlier evidence of mortality benefits creating turbulence among different stakeholders of screening programmes. Such differential evidence and emerging controversies have sparked heated debates challenging some conventional global views on mammographic breast screening.

History of screening mammography: How different countries of the world embarked on mammographybased breast screening programmes

Benefits of using mammography for early detection of breast cancer was researched as early as in the mid-twentieth century. The first randomized trial programme on breast cancer screening which was called the "Health Insurance Plan of New York City", was commenced in 1963 by Shapiro, Strax, and Venet. The results of this trial which evaluated benefits of mammography and clinical breast examination were first reported in 1966 followed by updates in 1971 and 1982 which showed a reduction of breast cancer mortality 
rates in the intervention group over controls [2].

Considering positive evidence on the benefits of screening mammography, the Canadian task force initiated their national breast cancer screening programme in 1976 [1]. In 1986, "Europe against Cancer" programme was launched with a population-based screening in a target group of 50 to 59-year females per reported mortality benefits in mammography of age specific groups [3]. The Forrest report (1986) [4], which led to wide acceptance of screening mammography in UK, mainly considered evidence form two RCTs of that time, the Health Insurance Plan of New York City [2] and the Swedish two county study [5]. The report suggested that screening would reduce breast cancer mortality by almost one third with more benefits than the costs involved [4]. Costs and benefits were considered in terms of quality adjusted life years (QALYs) and overdiagnosis was not considered an obstacle but follow up was suggested to find out its magnitude as the Swedish trial suggested overdiagnosis of $20 \%$ [4]. The Forrest report resulted in the introduction of population screening mammography in the UK. Australia started breast cancer screening of women aged 50 to 69 years only during the early 1990s [6]. The continent of Asia has relatively lower the number of breast cancer deaths compared to the rest of the world [7]. However, upper-middle income Asian countries such as Malaysia and Taiwan initiated population-based mammographic screening (since 2004) considering the rising incidence and death rates due to breast cancer in those countries [8]. Japan began clinical breast examination in 1987 , followed by recommendations to use mammography in combination with clinical breast examination as a populationbased program in the year 2000 [9].

In most countries, the initial screening programs included a combination of mammography with clinical or self-breast examination [9]. Nevertheless, the World Health Organization (WHO) guidelines do not recommend selfbreast examination as an effective method of breast cancer screening [10].

\section{Changes in breast cancer incidence and mortality in the screening era}

\section{- Rising incidence of breast cancer}

Incidence of breast cancer increased by nearly 30\% worldwide between the periods of 1973 to 1997 with some variations in the pattern of increase among the regions [7]. A rise in incidence was observed in North America in the 1980s, which slowed down during the mid-1990s, while a continued marked rise was seen in rest of the world including developing countries since mid1980s [7]. Asian countries, despite being among the regions with the lowest breast incidence, showed the highest rise in breast cancer incidence with most marked rates seen in Japan amounting to 78\% [7]. The rise in incidence in breast cancer in the western countries is largely attributed to detection of more and more small localized cancers by screening mammography [7], expressed as an 'artefactual increase' [11]. Some authors have sceptically expressed this association, which is common to many other cancers with specific screening programmes, 'as if screening was causing cancer' because a paralleled rise in incidence was not seen in control groups in randomized trials [1]. This rise in breast cancer incidence was marked among women of perimenopausal age group, who were the main participants at mammographic screening programmes.

Unlike in developed Western countries, factors such as westernisation of lifestyle and affluent living standards [12] have been counted for the rise in incidence in Asian countries, since a major contribution from screening was unlikely in the absence of population screening except in countries like Japan [7].

\section{- Changes in breast cancer mortality}

Despite rising incidence in breast cancer between 1973 to 1997, worldwide breast cancer mortality remained stable or decreased during the same period [7]. From the year 1989 to 1993 , age standardized breast cancer mortality rates dropped by $6.8 \%$ in US white women of all ages (13), while in the UK breast cancer death rates declined by $22 \%$ in both 20 to 49 year and 50 to 69- year age groups from 1987 to 1997 [11]. This decline in mortality in US and UK was considered statistically too rapid to be a sole effect of mammography and was attributed to result from both screening mammography and improvements in cancer therapy $[11,13,14]$.

\section{- Harms of screening mammography}

Evidence on the harms of screening mammography has been brought up and increasingly highlighted by many researchers over the past decades raising concerns both among the public and the policymaking authorities.

\section{- False positive screening tests}

A false positive screening test results when a woman is recalled for assessment following an abnormal screening mammogram but is proven cancer-free following assessment with no evidence of cancer up to one year. False positive rates vary between countries. The highest rates are reported in the US where the recall rate of 50 to 54 -year age group reached $13 \%-14 \%$ after the first mammogram [15]. More than $90 \%$ of such recalled mammograms are eventually declared cancer free [1]. 
However, assessment and follow up of these abnormal mammograms add a burden to the health budget. The cost for follow-up of these false positives is reported to be as high as $33 \%$ of the cost of breast screening [16].

More recently, patient anxiety triggered by false positive mammograms, identified as a notable harmful outcome of screening mammography, has drawn much attention of the scientific community and the public [1]. In addition to mere anxiety, other negative psychological attributes such as worry, sadness, sleep issues, impact on sexuality have been observed in these women identifying a significant psychological burden resulting from false positive mammograms $[17,18]$. Even though the false positive rates decline with subsequent screenings [19], the overall chance of a woman being recalled during 10 years of annual screening in US can be as high as 50\% [16], which further highlights the chance of women being subjected to undue stress by screening. The worry of having a missed cancer is observed to remain in some women even after the mammogram is cleared cancer free, amounting to sustained stress $[1,17]$.

\section{- Overdiagnosis and overtreatment of breast cancer}

Overdiagnosis is the detection of cancer which, if not for screening, would not have clinically manifested during the lifespan of a patient. It is a negative attribute common to many other specific cancer screening programmes as well [1]. Overdiagnosis leads to overtreatment including surgery and radiotherapy. Radiotherapy has been postulated to cause increased mortality in the screened group of women [17]. Varying rates have been estimated for overdiagnosis form population screening mammography by different research groups (ranging from 0 to $54 \%$ in different age groups), however, its exact magnitude is uncertain [10]. Marmot et al. [20], and Paci et al.[21] have estimated that for every one or two cases of overdiagnosis, at least one death due to breast cancer was prevented suggesting a balance between harms and benefits of population screening mammography [10].

\section{Debates and recent controversies on mammographic breast screening}

Although it has been widely accepted over decades that mammographic screening leads to reduced breast cancer mortality both in the invited for screening and screened populations, more recently some authors have challenged these results questioning the very foundation of mammographic screening.

The Canadian national breast screening study (1980 to 1996) was the first to report no mortality benefits in the intervention group over controls with screening mammography both in 40 49 year and in the 50 to 59-year females [22]. This study, which caused an uproar, was however criticized by many as not suitable for evidence based conclusions due to considerable drawbacks such as poor randomization, not excluding women with physical examination findings such as palpable lumps, palpable axillary nodes and nipple retraction during screening trials, use of poor-quality mammography images and inadequate statistical rigor [23]. It has also been suggested that screening mammography may increase mortality due to increased use of radiotherapy for management of screening detected small tumours [17].

Olsen \& Gøtzsche's Cochrane review has led to much controversy with their recommendation to abandon breast cancer screening concluding that there is a lack of reliable evidence on the claimed mortality benefits [17]. They also declared that breast cancer mortality was a biased factor towards mammographic screening. The review was heavily condemned by many as the reviewers discarded the majority of the randomized control trials available at the time, stating that the excluded studies were inadequately randomized but ignoring flaws in the two studies with no mortality benefits of mammography and for using cancer registry data instead of patient records (8). Furthermore, the Cochrane review considered a total number of invited women for breast cancer screening to be the participant numbers, which could generate inconclusive evidence on overdiagnosis rate. The review also gave increased attention to the harms of screening mammography. It was highlighted that when one breast cancer is diagnosed, ten females are overdiagnosed of having cancer resulting in overtreatment suggesting a $30 \%$ overdiagnosis rate [17].

\section{Decisions made following controversies: Current perspective on breast cancer screening}

Despite differential views on detection methodology, breast cancer remains the commonest cancer among women. It is also the second commonest cancer worldwide after lung cancer [24]. Of the total new cancer cases globally, 11.6\% were breast cancer and accounted for $6.6 \%$ of all cancer death rates in the year 2018 [24].

In light of new conflicting evidence on benefits and harmful effects of population-based screening mammography, many countries conducted their discussions regarding the use of screening mammography with panels of expertise in the area revisiting their national guidelines.

United Kingdom panel of experts concluded that out of women aged between 50 to 69 years who are invited for screening, 129 per 10,000 screened would have overdiagnosis (when estimated with a 19\% cumulative incidence of overdiagnosis) [20]. They also reported that of 100 women screened and 2000 women invited for screening, one breast cancer death was prevented: The panel concluded a $20 \%$ 
reduction in mortality from breast cancer among women who were invited for screening, taking into consideration 11 RCTs on breast cancer [20]. Therefore, a decision was made to continue population-based breast cancer screening in UK. Swiss Medical Board (Switzerland) in 2014 advised against screening mammography considering principally Olsen and Gøtzsche's Cochrane review evidence of inconclusive mortality benefits [25]. Nevertheless, a leading cancer association in Switzerland, "La Ligue Suisse Contre le Cancer" criticized the conclusion in Cochrane review to abandon population-based screening completely and recommended implementing quality control measure to uplift their existing practice [25].

It is agreed by many panels that the applicability of old clinical trial findings to the current screening practice remains uncertain [20]. Changes are made regarding the screening interval in countries considering the risks including radiation, cost and availability of resources. There continues to be a difference in opinions on the age at which screening should commence. WHO position paper on mammography screening 2014 concludes that mammography is the only method so far proven to be effective for organized mass screening for breast cancer and that there is a $20 \%$ reduction in mortality across all screened age groups in a well-planned population screening programme [10]. They also state that currently, the magnitude of the harms of screening mammography remains uncertain and that there appears to be a narrow trade-off between harms and benefits in the youngest (40-49 years) and older most (70-75 years) age groups [10]. Also understood is that early detection of breast cancer depends not only on screening mammography but also on many other factors such as breast cancer awareness and clinical breast examination carried out by a trained professional. At present, early recognition of breast cancer symptoms by patients, improved treatment methods and multidisciplinary approach to breast cancer treatment also have contributed to breast cancer mortality reduction, with mammography [26].

\section{Should low- middle income countries start population screening; Sri Lankan perspective on breast cancer screening}

\section{- Breast cancer burden in Sri Lanka}

Breast cancer is the commonest cancer next to oral cancer in Sri Lanka, with 3091 new cases diagnosed during 2018 (13.1\% of newly diagnosed breast cancers out of the total) [27]. Breast cancer was ranked as the second leading cause of cancer deaths after lung cancer in Sri Lanka [27]. Over the past decade (from 2001 to 2010) a 1.4-fold rise in the incidence of breast cancer was recorded in an island-wide study indicating a rising disease burden [28].

\section{- Applicability of population-based screening to middle} income countries

WHO breast cancer screening guidelines state that national breast cancer screening programmes are costeffective for upper-middle income countries as opposed to the low-middle income countries [10]. Population screening mammography is considered non-cost effective for limited resource settings which include low and lowmiddle income countries [10].

\section{- A place for opportunistic screening}

Currently, there are opportunistic mammography screening facilities in a limited number of centres in Sri Lanka which are mostly distributed in the private sector. NCCP guidelines recommend screening women aged between 50 to 69 years [29] which is in concurrence with the current rising breast cancer incidence seen in the women over 50 years [28]. How well these guidelines are reached and practised by the individual centres remain unexplored.

It is imperative to note that WHO breast cancer screening guidelines [10] are brisk to remind that opportunistic screening can tip off the tight balance between benefits and harms of mammography screening towards the harms, especially if such opportunistic programmes are not properly organized and controlled. Validated protocols, para-clinical staff experienced in communication with patients and quality assurance are some of the key features that may need to be strengthened in individual opportunistic screening centres in Sri Lanka among many other criteria specified by the WHO.

Early recognition of breast cancer and timely interventions is considered the most important aspect in the reduction of breast cancer deaths [10]. Therefore, considering the resources available, efforts should be directed at increasing breast cancer awareness especially among the postmenopausal age group, promotion of accurate clinical breast examination and optimising diagnostic facilities, staging and improved access to treatment [30].

All authors disclose no conflict of interest. The study was conducted in accordance with the ethical standards of the relevant institutional or national ethics committee and the Helsinki Declaration of 1975, as revised in 2000 .

\section{References}

1. Fletcher SW. Breast cancer screening: a 35-year perspective. Epidemiologic reviews. 2011;33(1):165-75. https://doi.org/10.1093/epirev/mxr003.

2. Shapiro S, Venet W, Strax P, Venet L, Roeser R. Ten-to fourteenyear effect of screening on breast cancer mortality. Journal of the National Cancer Institute. 1982;69(2):349-55. https://doi.org/10.1093/jnci/69.2.349. 
3. Biesheuvel C, Weige S, Heindel W. Mammography screening: evidence, history and current practice in Germany and other European countries. Breast Care. 2011;6(2):104-9. https://doi.org/10.1159/000327493.

4. Forrest P. Breast cancer screening: report to the Health Ministers of England, Wales, Scotland \& Northern Ireland: hmso; 1986.

5. Tabar L, Gad A, Holmberg L, Ljungquist U, Group KCP, Fagerberg C, et al. Reduction in mortality from breast cancer after mass screening with mammography: randomised trial from the Breast Cancer Screening Working Group of the Swedish National Board of Health and Welfare. The Lancet. 1985;325(8433):829-32.

6. Olver IN, Roder D. History, development and future of cancer screening in Australia. Public Health Res Pract. 2017;27(3):2731725. https://doi.org/10.17061/phrp2731725.

7. Althuis MD, Dozier JM, Anderson WF, Devesa SS, Brinton LA. Global trends in breast cancer incidence and mortality 1973-1997. International journal of epidemiology. 2005;34(2):405-12. https://doi.org/10.1093/ije/dyh414.

8. Sitt J, Lui C, Sinn L, Fong J. Understanding breast cancer screening - past, present, and future. Hong Kong Med J. 2018;24(2):166-74. DOI: 10.12809/hkmj177123.

9. Hamashima C, Guidelines JRGftDoBCS, Hamashima C C, Hattori M, Honjo S, Kasahara Y, et al. The Japanese guidelines for breast cancer screening. Japanese journal of clinical oncology. 2016;46(5):482-92.

https://doi.org/10.1093/jjco/hyw008.

10.Organization WH. WHO position paper on mammography screening: World Health Organization; 2014.

11.Peto R, Boreham J, Clarke M, Davies C, Beral V. UK and USA breast cancer deaths down $25 \%$ in year 2000 at ages 20-69 years. Lancet. 2000;355(9217):1822.

12.Porter PL. Global trends in breast cancer incidence and mortality. Salud publica de Mexico. 2009;51:s141-s6.

https://www.scielosp.org/scielo.php?pid=S0036$36342009000800003 \&$ script $=$ sci abstract.

13. Chu KC, Tarone RE, Kessler LG, Ries LA, Hankey BF, Miller $\mathrm{BA}$, et al. Recent trends in US breast cancer incidence, survival, and mortality rates. JNCI: Journal of the National Cancer Institute. 1996;88(21):1571-9. https://doi.org/10.1093/jnci/88.21.1571.

14.Beral V, Hermon C, Reeves G, Peto R. Sudden fall in breast cancer death rates in England and Wales. The Lancet. 1995;345(8965):1642-3.

https://doi.org/10.5555/uri:pii:S0140673695901515.

15.Smith-Bindman R, Chu PW, Miglioretti DL, Sickles EA, Blanks $\mathrm{R}$, Ballard-Barbash $\mathrm{R}$, et al. Comparison of screening mammography in the United States and the United Kingdom. Jama. 2003;290(16):2129-37. 10.1001/jama.290.16.2129.

16.Elmore JG, Barton MB, Moceri VM, Polk S, Arena PJ, Fletcher SW. Ten-year risk of false positive screening mammograms and clinical breast examinations. New England Journal of Medicine. 1998;338(16):1089-96.

https://doi.org/10.1056/NEJM199804163381601.
17.Olsen O, Gøtzsche PC. Screening for breast cancer with mammography. Cochrane Database of Systematic Reviews. 2001(4). https://doi.org/10.1002/14651858.CD001877.

18.Brodersen J, Siersma VD. Long-term psychosocial consequences of false-positive screening mammography. The Annals of Family Medicine. 2013;11(2):106-15.10.1370/afm.1466

19.Coldman AJ, Phillips N. False-positive screening mammograms and biopsies among women participating in a Canadian provincial breast screening program. Canadian Journal of Public Health. 2012;103(6):e420-e4. 10.1007/BF03405630.

20.Marmot MG, Altman D, Cameron D, Dewar J, Thompson S, Wilcox M. The benefits and harms of breast cancer screening: an independent review. British journal of cancer. 2013;108(11):2205.

21.Paci E. Summary of the evidence of breast cancer service screening outcomes in Europe and first estimate of the benefit and harm balance sheet. Journal of medical screening. 2012;19(1_suppl):5-13. https://doi.org/10.1258/jms.2012.012077.

22.Miller AB, Study-2 FtCNBS, To T, Study-2 FtCNBS, Baines CJ, Study-2 FtCNBS, et al. Canadian National Breast Screening Study-2: 13-Year Results of a Randomized Trial in Women Aged 50-59 Years. JNCI: Journal of the National Cancer Institute. 2000;92(18):1490-9. 10.1093/jnci/92.18.1490.

23.Heywang-Köbrunner SH, Schreer I, Hacker A, Noftz M, Katalinic A. Conclusions for mammography screening after 25year follow-up of the Canadian National Breast Cancer Screening Study (CNBSS). European radiology. 2016;26(2):342-50.

24.Bray F, Ferlay J, Soerjomataram I, Siegel RL, Torre LA, Jemal A. Global cancer statistics 2018: GLOBOCAN estimates of incidence and mortality worldwide for 36 cancers in 185 countries. CA: a cancer journal for clinicians. 2018;68(6):394424. https://doi.org/10.3322/caac.21492.

25.Chiolero A, Rodondi N. Lessons from the Swiss Medical Board recommendation against mammography screening programs. JAMA internal medicine. 2014;174(10):1541-2. 10.1001/jamainternmed.2014.4197.

26.Martínez-Alonso M, Carles-Lavila M, Pérez-Lacasta MJ, PonsRodríguez A, Garcia M, Rué M. Assessment of the effects of decision aids about breast cancer screening: a systematic review and meta-analysis. BMJ open. 2017;7(10):e016894. 10.1136/bmjopen-2017-016894

27. IARC. Sri Lanka: Incidence, mortality and prevalence by cancer. 2018. http:/gco.iarc.fr/today/data/factsheets/populations/144sri-lanka-fact-sheets.pdf.

28. Fernando A, Jayarajah U, Prabashani S, Fernando EA, Seneviratne SA. Incidence trends and patterns of breast cancer in Sri Lanka: an analysis of the national cancer database. BMC cancer. 2018;18(1):482. https://doi.org/10.1186/s12885-0184408-4.

29. Early detection and management of breast symptoms, National Guideline for Primary Care Doctors \& Family Physicians National cancer control programme 2014.

30. Guide to cancer early diagnosis. World Health Organization 2017. 